\title{
STELLAR LUMINOSITY AND MASS FUNCTIONS OF GLOBULAR CLUSTERS ${ }^{1}$
}

\author{
GIAMPAOLO PIOTTO \\ Dipartimento di Astronomia, Università di Padova \\ Vicolo dell'Osservatorio 5, I-35122 Padova, Italy \\ AND \\ ADRIENNE M. COOL AND IVAN R. KING \\ Astronomy Department, University of California \\ Berkeley, CA 94720-3411, U.S.A.
}

\begin{abstract}
HST makes it possible for the first time to study nearly the entire mass range of globular-cluster main sequences, from the turnoff down almost to the theoretical limit for hydrogen ignition. We present mainsequence luminosity functions (LFs) for four clusters that include stars with $\mathcal{M}<0.15 \mathcal{M}_{\odot}$ in all cases. We compare these and other LFs that have been obtained with HST for a total of five globulars to date. Two of the three clusters in the sample that have similar metallicities have nearly identical LFs, while the third is relatively deficient in low mass stars. Possible implications of this finding are briefly discussed. Inferred mass functions vary significantly depending on the mass-luminosity relations that are adopted.
\end{abstract}

\section{Introduction}

The Hubble Space Telescope (HST) allows photometry and counting of globular-cluster (GC) stars several magnitudes fainter than is possible with ground-based equipment. For the closest clusters, luminosity functions (LFs) and the resulting mass functions (MFs) can be extended down to $\sim 0.10 \mathcal{M}_{\odot}$, or nearly the hydrogen-burning limit. The MFs of GC stars provide impor-

\footnotetext{
${ }^{1}$ Based on observations with the NASA/ESA Hubble Space Telescope, obtained at the Space Telescope Science Institute, which is operated by AURA, Inc., under NASA contract NAS5-26555.
} 
tant observational inputs into a wide variety of astrophysical problems, including (1) the realistic dynamical modeling of individual clusters; (2) the role of dynamical evolution in modifying globular-cluster MFs; (3) the amount of mass contained in very-low-mass (VLM) and brown-dwarf stars in globulars and, by extension, in the halo; (4) globular-cluster formation and initial MFs.

The first extensive comparison of Galactic GC MFs was presented by Scalo (1986), but was still based on star counts on photographic plates. Due to the limitation in mass range and to the big uncertainties in the incompleteness at the low-mass end, Scalo concluded there was no compelling evidence for differences among GC MFs. This result was questioned in the same year by McClure et al. (1986), who presented the first set of LFs based on CCD photometry. From a sample of 7 GCs, they noted a strong dependence of the slope of the MF on the metal content, the steepness increasing with decreasing metallicity. In a subsequent comparison of 17 GCs, Piotto (1991) suggested that dynamical evolution could also be important. A detailed multivariate statistical analysis (Djorgovski, Piotto, \& Capaccioli 1993) confirmed both these results, and showed that the MFs depend mainly on a cluster's position in the Galaxy, and only secondarily on metallicity: clusters closer to the Galactic plane and center have flatter MFs. (The secondary dependence on metallicity is in the same direction as originally suggested by McClure et al.) The main limitation of all these ground-based studies is the small mass range $\left(0.5<\mathcal{M} / \mathcal{M}_{\odot}<0.8\right)$ and the fact that the MFs were approximated by power laws. In the same period, Richer et al. (1991) tried to push the study of MFs to significantly fainter magnitudes in 6 globulars. Their results suggested that the MFs of at least some globulars are so steep at the faint end that VLM stars make a significant contribution to the total cluster mass. Extending this finding to the halo MF, the authors suggested that VLM stars could contribute to the missing mass of the Galactic halo.

Here we present LFs measured with the HST/WFPC2 for four globular clusters: M30 (NGC 7099), M15 (NGC 7078), 47 Tuc (NGC 104), and NGC 6397. We compare our results to existing HST studies of the latter three of these GCs, to the HST study of $\omega$ Cen by Elson, Gilmore, \& Santiago (1995), and to earlier ground-based studies. We discuss the main properties of the LFs for the clusters of our sample, and infer the corresponding MFs using the most recently calculated mass-luminosity relations.

\section{The Data Set}

All the images were taken with the WFPC2 in parallel mode, at $\sim 4.6$ arcmin from the cluster centers. Filters and total exposure times are given 
TABLE 1. Data Set

\begin{tabular}{|c|c|c|c|c|c|c|}
\hline Cluster & $(m-M)_{I}$ & {$[\mathrm{Fe} / \mathrm{H}]$} & Filter & $\begin{array}{r}\text { Exp time } \\
{[\mathrm{sec}]}\end{array}$ & $M_{I, 50 \%}$ & $\begin{array}{l}\mathcal{M}_{\text {lim }} \\
{\left[\mathcal{M}_{\odot}\right]}\end{array}$ \\
\hline NGC 6397 & 12.05 & -1.9 & $\begin{array}{l}\text { F555W } \\
\text { F814W }\end{array}$ & $\begin{array}{l}14200 \\
18700\end{array}$ & 12.3 & 0.10 \\
\hline NGC 7078 & 15.26 & -2.2 & $\begin{array}{l}\text { F606W } \\
\text { F814W }\end{array}$ & $\begin{array}{l}6050 \\
6050\end{array}$ & 10.7 & 0.11 \\
\hline NGC 7099 & 14.48 & -2.1 & $\begin{array}{l}\text { F555W } \\
\text { F814W }\end{array}$ & $\begin{array}{r}15300 \\
8700\end{array}$ & 10.5 & 0.12 \\
\hline 47 Tuc & 13.35 & -0.7 & $\begin{array}{l}\text { F606W } \\
\text { F814W }\end{array}$ & $\begin{array}{l}1600 \\
2000\end{array}$ & 10.0 & 0.13 \\
\hline
\end{tabular}

in Table 1, together with metallicities and adopted distance moduli. The reduction procedures are described by Cool \& King (1995) and Cool, Piotto, \& King (1995). Instrumental magnitudes were transformed into the WFPC2 "ground" system following Holtzman et al. (1995). Here we present only LFs from the $V_{555}$ and $I_{814}$ photometry, which are very similar to Johnson standard $V$ and $I$; the $V_{606}$ filter is significantly different from Johnson $V$.

\section{The Luminosity Functions}

Particular attention has been devoted to determination of the completeness and to corrections for field-star contamination (see Piotto, Cool, \& King 1995). The LFs presented here include only points for which the completeness was found to be $>50 \%$. With the exception of 47 Tuc, which has the most crowded images, the completeness drops below $80 \%$ only in the last $\simeq 1$ mag of any of the LFs. The last two columns in Table 1 give the absolute $I_{814}$ magnitude at which $50 \%$ completeness was reached, and the approximate corresponding mass. All the LFs have been obtained from color-magnitude diagrams (CMD), which allow the direct discrimination of cluster stars from background/foreground objects. Field-star contamination was significant only in the case of NGC 6397, which has the lowest Galactic latitude of the four clusters $\left(b=-12^{\circ}\right.$ ). (Compare the NGC 6397 CMD [Fig. 1 in Cool, Piotto, \& King] with that of M30 [Fig. 2 in King], both elsewhere in these proceedings.)

In Figure 1 we present the $I_{814}$ LF for NGC 6397. The star numbers peak at $I_{814} \sim 20.5\left(V_{555} \sim 22.2\right)$, and then decline by a factor of 3 over the next three magnitudes. Also shown is the HST-based LF of Paresce, De Marchi, \& Romaniello (1995). The two HST-based LFs span somewhat 


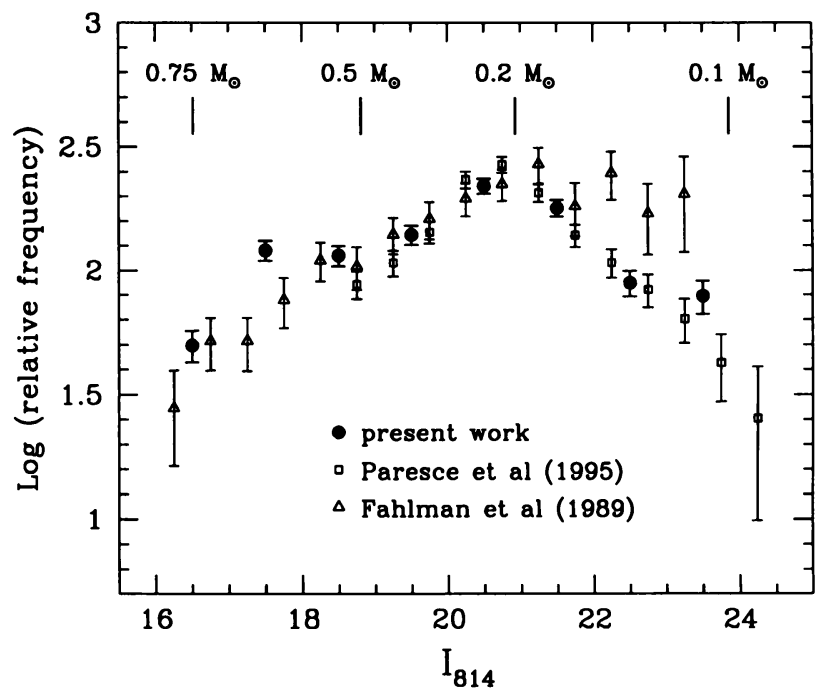

Figure 1. Two HST LFs for NGC 6397, along with the ground-based LF of Fahlman et al. (1989).

different magnitude ranges. Ours extends closer to the turnoff, owing to the inclusion of short exposures in our observing program, and ends with a bin at $I_{814}=23-24\left(V_{555} \simeq 25.3-26.5\right)$, beyond which we consider the field-star corrections to be too uncertain to permit a reliable measurement of the LF (see the color-magnitude diagram in Fig. 1 of Cool, Piotto, \& King elsewhere in this volume). Over the common range, the two HST-based LFs are in good agreement. Also shown is the ground-based $I$-band LF of Fahlman et al. (1989), which for $I_{814} \leq 21.5$ is in reasonable agreement with the HST-based LFs. At fainter magnitudes however, the ground-based LF rises significantly above both of the presumably more reliable HST-based LFs. Similar discrepancies between HST and ground-based results at the faint end of the LF were noted by Elson et al. (1995) for $\omega$ Cen.

In Figure 2 we compare the $I_{814}$ and $V_{555}$ HST LF with the groundbased $I$ LF (Drukier et al. 1993), and V LF (Piotto, Ortolani, \& Zoccali 1995) obtained at similar distance from the cluster center. The agreement is quite good, suggesting that while ground-based LFs should not be relied on at very faint magnitudes, they can be relied on at brighter magnitudes. This is important, as it means that the ground-based LFs can be usefully applied to extend the HST LFs to bright magnitudes (up to the turnoff), for which the use of HST would both be inefficient and unnecessary. In what 


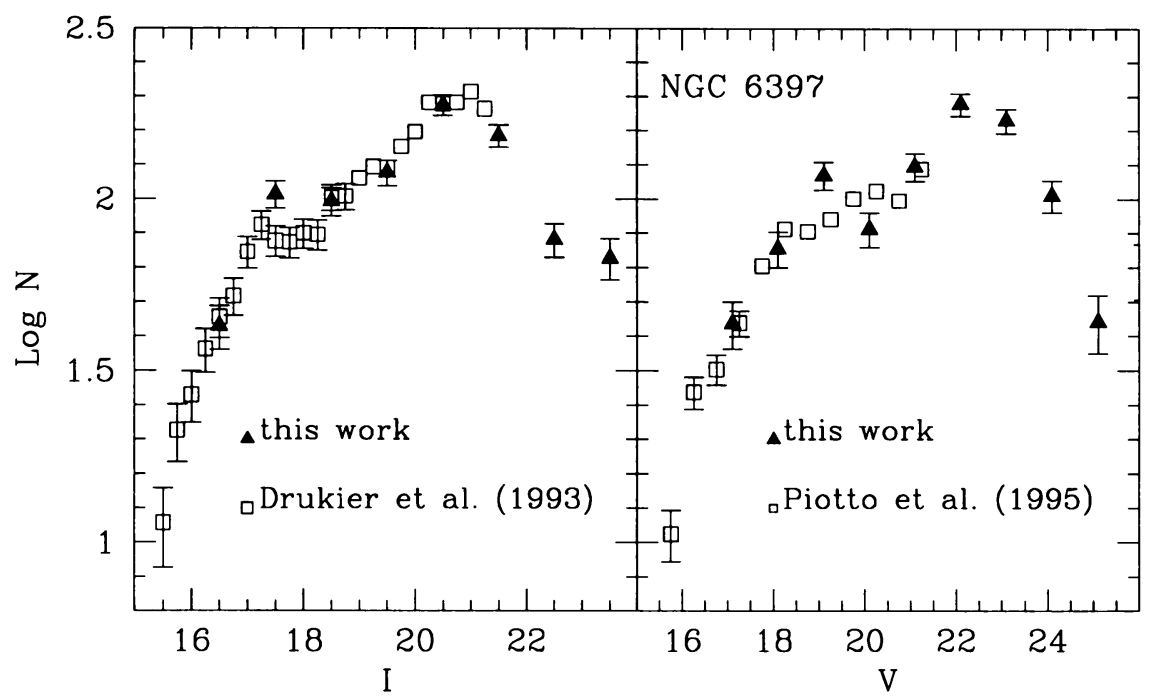

Figure 2. The $I_{814}$ and $V_{555}$ HST LFs for NGC 6397 are compared with the ground-based LFs by Drukier et al (1993) and Piotto et al (1995), obtained at similar distances from the cluster center. Where omitted, the error bars are smaller than the symbol size.

follows, the NGC $6397 \mathrm{HST}$ LF, which is truncated at $M_{555}=4.7\left(M_{814}\right.$ $=4$ ) due to the saturation of bright stars even in the short exposures, has been extended to the turnoff using the ground-based LFs shown in Fig. 2.

The $I_{814}$ and $V_{555}$ LFs for the three metal-poor clusters (NGC 6397, $\mathrm{M} 15$, and M30) are compared in the left and right panels of Figure 3. (M15 does not appear in the right panel, as the $V$ data were taken with a different filter.) Vertical shifts were made to bring the LFs into alignment according to a least-squares algorithm, in the magnitude intervals $4.0<M_{814}<7.0$ and $4<M_{555}<7.0$. As shown by King elsewhere in this volume, the measured (local) LF of NGC 6397 closely resembles the cluster's global LF. As M30 and M15 are structurally very similar to NGC 6397 , and the observations were similarly taken well outside of their cores, their observed LFs should also closely resemble the global LFs. What then makes these three clusters a particularly advantageous comparison is that their metal content is very similar (see Table 1, Col. 3), which implies that the massluminosity relations (MLR) for their stellar populations will also be very similar. Similarities or differences in their MFs can thus be discerned in a direct comparison of their observed LFs.

The broad features of the three $I_{814}$ LFs are similar. Each rises steadily to a peak near $M_{814} \simeq 8.5-9.0$, then bends over and drops significantly to the limit of the observations. But when the LFs are overlaid and lined up in the range $M_{814}=4.0-7.0$ (left panel), it becomes clear that while the LFs 


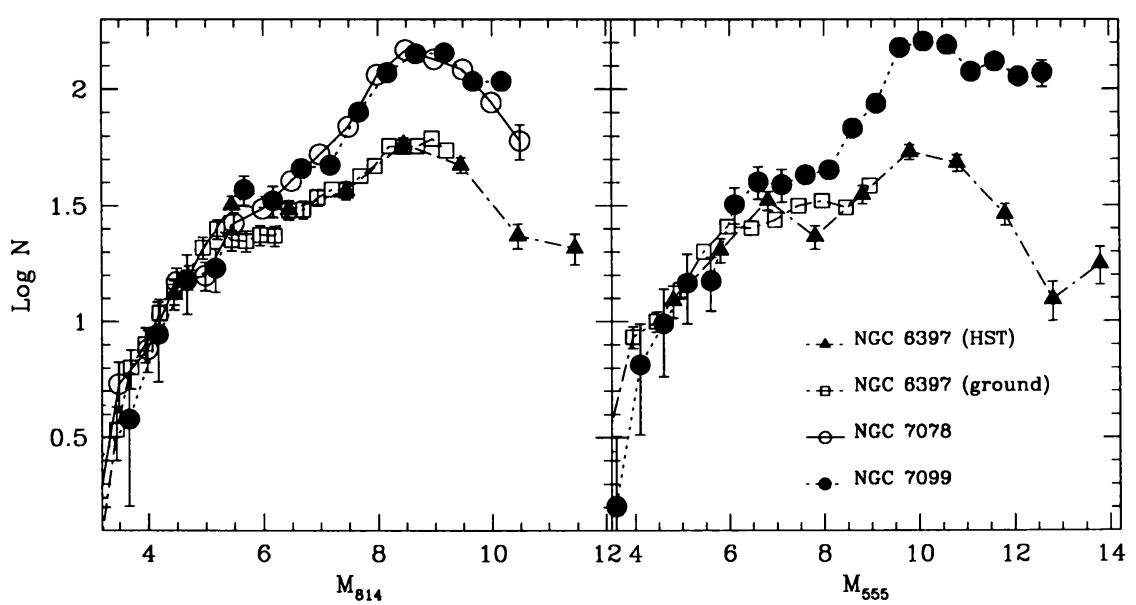

Figure 3. $I_{814}$ (left panel) and $V_{555}$ (right panel) LFs for the three metal-poor clusters, converted to absolute magnitudes. The LF for NGC 6397 has been extended to bright magnitudes (up to the TO), using ground-based LFs (cf. Fig. 2). Where omitted, the error bars are equal to or smaller than the symbol size.

of M30 and M15 are indeed very similar, NGC 6397 is markedly deficient in faint stars. A similar conclusion is reached by comparing the $V_{555} \mathrm{LF}$ of NGC 6397 with that of M30 (right panel). The differences are statistically highly significant, being many times the uncertainties in the single points of the LFs.

Interestingly, the difference between NGC 6397 and the near-twin LFs of M30 and M15 becomes apparent only when the LFs are compared over a wide range of magnitudes. De Marchi and Paresce (1995a) analyzed the same M15 data set that we have analyzed, and obtained an LF that is quite similar to ours. However, when they compared their M15 LF to that of Paresce et al. (1995) for NGC 6397, the two appeared indistinguishable. The resolution of this apparent contradiction between our respective results is that their comparison was limited to magnitudes in the interval $M_{814} \simeq$ 6.5-10. The brighter magnitudes included in our NGC 6397 measurements allow a more stringent comparison, from $M_{814}=4.5-10.5$. The relative paucity of faint stars in NGC 6397 then becomes unmistakable, and is further confirmed by the extension of the NGC $6397 \mathrm{LFs}$ to even brighter magnitudes using ground-based data.

The similarity of the LFs of M15 and M30 over a span of more than 6 magnitudes is striking. It implies that their MFs are very similar in the range of approximately $0.12<\mathcal{M} / \mathcal{M}_{\odot}<0.8$. It would appear that either these two clusters were born with similar MFs and have changed little since, or that they were born similar and have changed similarly. A scenario 
in which they were born different and have evolved to become the same seems too contrived. In view of this similarity, it is natural to ask why NGC 6397, which has a similar metallicity and similar morphology (all three are post-core-collapse clusters), should have so many fewer low-mass stars. As further discussed by King elsewhere in these proceedings, the deficiency may be the result of tidal shocks, to which NGC 6397 is quite vulnerable, given its low-inclination orbit and small perigalacticon. Even without this dynamical explanation, the difference between these clusters is in qualitative agreement with the correlations between MF slopes and Galactic position identified by Djorgovski et al. (1993).

In Figure 4 we show the $I_{814}$ LF for 47 Tuc. The LFs of the metal-poor clusters from Fig. 3 are shown as lines, and the triangles represent the LF obtained by Elson et al. (1995) for $\omega$ Cen. The LFs have been aligned using a least-squares algorithm in the magnitude interval $5.0<M_{814}<7.0$. The 47 Tuc LF peaks at $M_{814} \sim 9$, at somewhat fainter magnitudes than the three metal-poor clusters, and then turns over. It agrees well with the LF obtained by De Marchi \& Paresce (1995b) for a different field in 47 Tuc at a similar distance from the cluster center. Omega Cen is the only cluster of the five for which no drop-off has been detected to the limit of the existing HST observations.

Since $\omega$ Cen and 47 Tuc are intermediate and metal-rich clusters, respectively, the MLRs appropriate for their stellar populations will be different from each other and from the metal-poor clusters. In addition, a non-trivial correction of the observed LFs may be required to convert to global LFs for these clusters. For both these reasons, it is inadvisable to draw any conclusions from the LF comparison in Fig. 4. The dynamical modeling required to convert the local LFs to global ones is under way, and will be presented elsewhere.

\section{The Mass Functions}

If the results of star counts are to be used in modeling globular clusters, and if clusters with differing metallicities are to be compared, we must transform the observed LFs to MFs. This transformation requires an appropriate MLR, and it is particularly sensitive to the latter, as the transformation depends on its derivative. Unfortunately, the MLRs for low-mass $\left(\mathcal{M}<0.5 \mathcal{M}_{\odot}\right)$ stars are quite uncertain: there are still serious difficulties in both the calculation of the stellar structure (critically dependent on the difficult evaluation of both the opacity and the equation of state for a cool gas) and in the treatment of their atmospheres (Alexander et al. 1995).

Several MLRs for low-mass stars now exist in the literature, some of which have appeared quite recently. Consensus has yet to be reached, ow- 


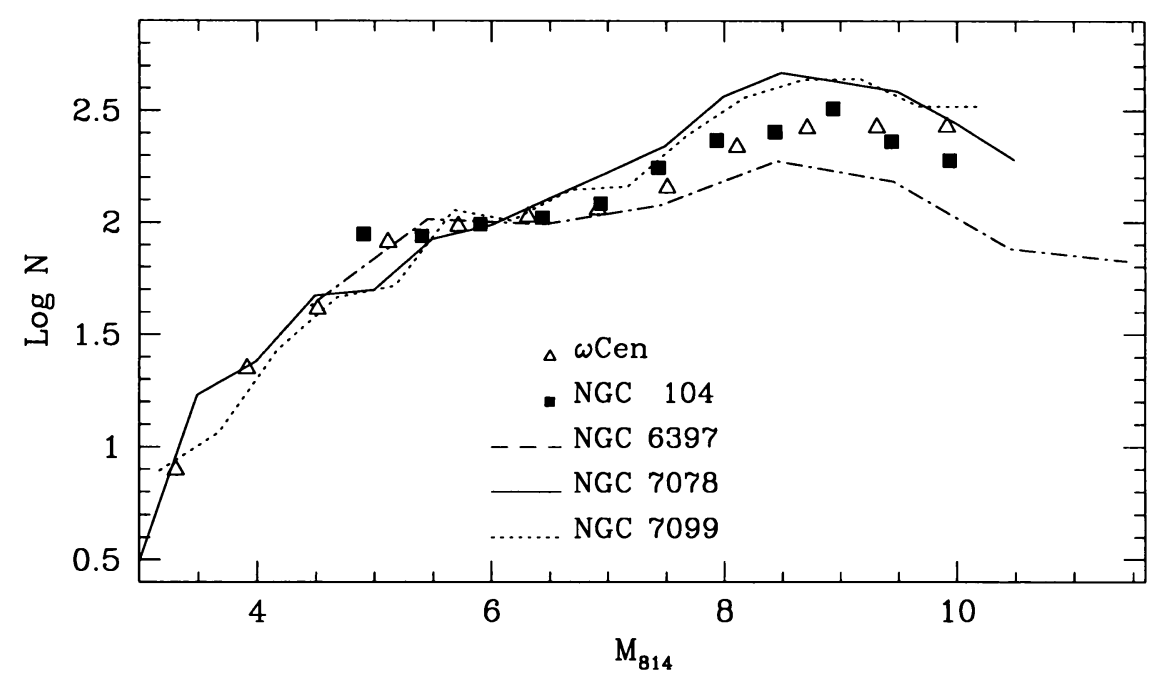

Figure 4. $M_{814}$ LFs for the 5 clusters in the sample discussed in this paper.

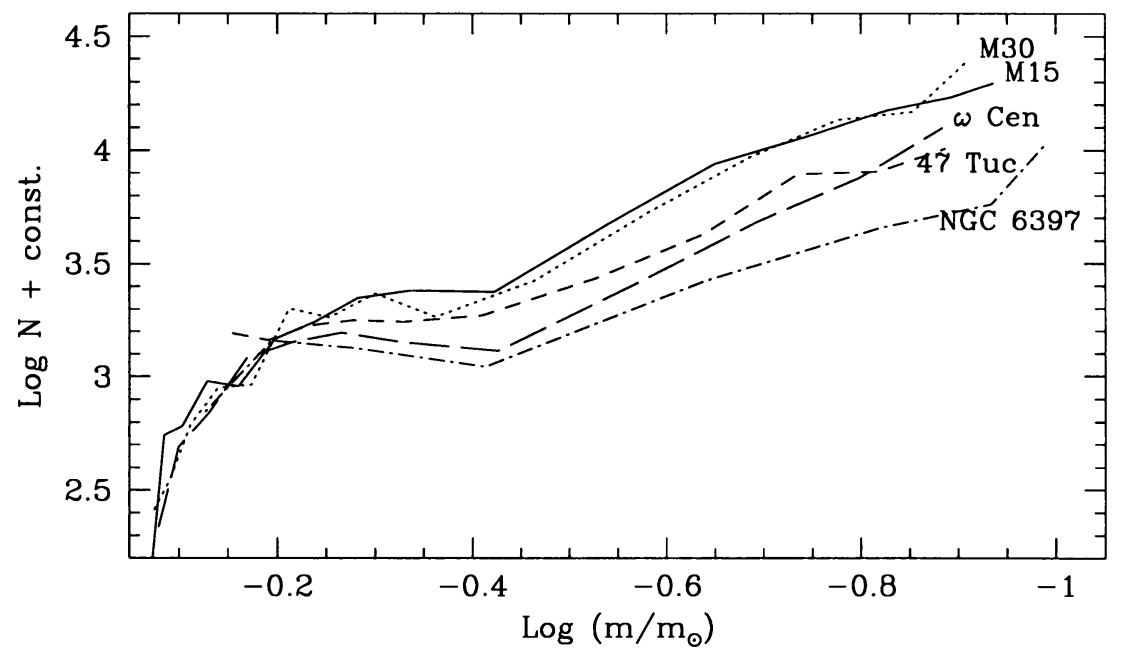

Figure 5. MFs derived from the LFs in Fig. 4, using the mass-luminosity relations of D'Antona \& Mazzitelli (1995), which yield the steepest slopes at very low masses.

ing in part to a paucity of observational constraints. These MLRs differ from one another in ways that potentially can have a significant impact on the MFs derived from them. In particular, differences in the MLR slopes for very-low-mass stars are sufficient to produce significant differences in the slopes of the resulting MFs. In an effort to determine how large these variations can be, we have experimented with several of the available MLRs: 
Baraffe et al. (1995), Bergbusch \& VandenBerg (1992), D'Antona \& Mazzitelli (1995), and Fahlman et al. (1989), as well as a set of models specifically calculated for the metallicity of these clusters by the Teramo group (Alexander et al. 1995).

In Figure 5 we show the MFs extracted from the $I_{814}$ LFs of Fig. 4, using the MLRs of D'Antona \& Mazzitelli (1995). Similar results were obtained by converting the $V_{555}$ LF to mass. This set of MFs represents one extreme, since these MLRs have the steepest (and most rapidly steepening) slope of any of the MLRs we tried, and thus produce the steepest MFs. All the resulting MFs rise steadily at the low-mass end. In the interval $0.1<$ $\mathcal{M} / \mathcal{M}_{\odot}<0.4$ the MFs can reasonably well be represented by power laws with slopes in the range $x=0.6-1.0$ (where for the Salpeter law $x=1.35$ ). The best fits to the MFs for NGC $6397(x=0.6)$ and $\omega$ Cen $(x=0.9)$ are both shallower than the MFs slopes found for these clusters in the ground-based study of Richer et al. (1991). None of the MFs obtained using D'Antona and Mazzitelli's (1995) MLR has a slope exceeding $x=1.0$, though M30 and M15 approach this limiting case, which would correspond to a marginally divergent cluster mass if the MF slopes can be extrapolated into the brown dwarf regime.

At the other extreme are the MFs we obtain by using the MLRs of Alexander et al. (1995), which have a shallower slope for very-low-mass stars. With these MLRs, the MFs have slopes in the range $x=-0.1$ (NGC 6397 ) to $x=0.7$ (M30), though the MFs deviate from power laws more than in the previous case. There is a tendency for the MFs to flatten out beyond $\log \left(\mathcal{M} / \mathcal{M}_{\odot}\right)=-0.8$ when these MLRs are used. In no case do we find evidence for a decline in the MF following the flattening.

\section{Conclusions}

With HST, main-sequence LFs can be readily measured from the turnoff down to less than $0.2 \mathcal{M}_{\odot}$ in a large number of clusters, allowing for much more detailed comparisons of cluster LFs and MFs than have previously been possible. In the closest clusters, observations have already reached nearly to the hydrogen-burning limit, and future observations should reach even farther. While a sample of five clusters is too small to draw any broad conclusions, the initial results of comparisons between clusters with similar metallicities are intriguing, and provide support for the view that clusters may be born with similar MFs, which can then be altered by interactions with the Galaxy. As the sample of clusters observed with HST increases, much more will be learned about the various factors influencing cluster MFs.

The new capacity afforded by HST in the study of globular clusters 
will provide important new constraints on increasingly detailed dynamical modeling of globulars, which will interact with computations such as those we have admired at this meeting. At the same time, it is clearer than ever that the information contained in the observational data will be fully exploited only when the inner and outer workings of very-low-mass stars are more thoroughly understood.

\section{Acknowledgments}

This work was supported by NASA grant NAG5-1607. We thank F. D'Antona, E. Brocato, V. Castellani, and S. Cassisi for providing isochrones in advance of publication, and P. Stetson for his generosity with software.

\section{References}

Alexander, D.R., Brocato, E., Cassisi, S., Castellani, V., Ciacio, F., \& Degl'Innocenti, S. 1995, A\&A, submitted

Baraffe, I., Chabrier, G., Allard, F., \& Hauschildt, P.H. 1995, ApJ, 446, L35

Bergbusch, P. A., \& VandenBerg, D. A. 1992, ApJS, 81, 163

Cool, A. C., \& King, I. R. 1995, in Calibrating HST: Post Servicing Mission, ed. A. Koratkar \& C. Leitherer (Baltimore: STScI), p. 290

Cool, A. M., Piotto, G., \& King, I. R. 1995, submitted to ApJ

D'Antona, F., \& Mazzitelli, I. 1995, ApJ, Dec. 1, in press

De Marchi, G., \& Paresce, F., 1995a, A\&A, in press

De Marchi, G., \& Paresce, F., 1995b, A\&A, in press

Drukier, G. A., Fahlman, G. G., Richer, H. B., Searle, L., \& Thompson, I. 1993, AJ, 106, 2335

Djorgovski, S., Piotto, G., \& Capaccioli, M. 1993, AJ, 105, 2148

Elson, R. A. W., Gilmore, G. F., \& Santiago B. X. 1995, AJ, 110, 682

Fahlman, G. G., Richer, H. B., Searle, L., \& Thompson, I. B. 1989, ApJ, 343, L49

Holtzman, J. A., Burrows, C. J., Casertano, S., Hester, J. J., Watson, A. M., \& Worthey, G. S. 1995, preprint

McClure, R. D., VandenBerg, D. A., Smith, G. H., Fahlman, G. G., Richer H. B., Hesser, J. E., Harris, W. E., Stetson, P. B., \& Bell, R. A. 1986, ApJL, 307, L49

Paresce, F., De Marchi, G., \& Romaniello, M. 1995, ApJ, 440, 216

Piotto, G. 1991, in Formation and Evolution of Star Clusters, ed. K. Janes, (San Francisco: ASP), p. 200

Piotto, G., Cool, A.M., \& King, I.R. 1995, preprint

Piotto, G., Ortolani, S., \& Zoccali, M. 1995, preprint

Richer, H. B., Fahlman, G. G., Buonanno, R., Fusi Pecci, F., Searle, L., \& Thompson, I. B. 1991, ApJ, 381, 147

Scalo J.M. 1986, Fund. of Cosmic Phys., 11, 1 